\title{
Research on the Value of Ultrasound Diagnosis in Thyroid Gland Lesions
}

\author{
Yan Liu, Xinying Li, Ruizhi Hou \\ China-Japan Union Hospital of Jilin University, Changchun, Jilin Province, 130021
}

Keywords: Value, Ultrasound Diagnosis, Thyroid Gland Lesions.

\begin{abstract}
Thyroid is the body's largest endocrine glands, the main function is used to synthesize, store and secrete thyroxine. Thyroid function interlinks with the activities of various organs and systems of the human body as well as the external environment. The main regulatory mechanisms include the hypothalamic-pituitary-thyroid axis controlled system and the autoregulation system in the thyroid gland. With the development of imaging, ultrasonography has become a diagnostic tool for thyroid-space lesions, which solves the difficulty of qualitative preoperative occupying lesions. This study selected 85 cases of thyroid gland lesions treated in China-Japan Union Hospital of Jilin University from 2011 to 2012 to investigate the clinical value of ultrasonic diagnosis of thyroid space-occupying lesions.
\end{abstract}

\section{Introduction}

Thyroid in the body's main function is the storage and synthesis and secretion of thyroid hormones, the metabolism of the human body have a significant impact [1]. Thyroid space occupying lesions are more common clinical conditions, is divided into benign and malignant lesions, the diagnosis of thyroid lesions often use surgical pathology diagnostic methods to identify. With the continuous development of medical technology, ultrasound in the diagnosis and differential diagnosis of thyroid disease has a good clinical effect. This time from February 2015 to August 2016 in our hospital for treatment of thyroid lesions of 80 patients for clinical research, all patients were diagnosed by ultrasound, ultrasound diagnosis of thyroid lesions of the clinical effect.

\section{Materials and Methods}

Eighty patients with thyroid space-occupying lesions treated in our hospital from February 2015 to August 2016 were selected as clinical analysis subjects. Among the 80 patients, 42 (52.5\%) were male and 38 (47.5\%) were female. The mean age was $48.6 \pm 10.7$ years (range, 23-78 years). The course of disease ranged from 3 to 20 years with a mean duration of $10.2 \pm 3.4$ years. The inclusion criteria for this study were: All patients who participated in the study were not tested for thyroid-related indicators prior to the study, and all patients signed the study for informed consent. Exclusion of patients aged $<20$ years or $>80$ years; Exclusion of thyroid hormone treatment, pathological examination, the results were inconclusive patients; In addition to the anatomy of the neck tissue abnormalities also excluded the study.

Eighty patients were diagnosed by color Doppler sonography using a color Doppler ultrasound diagnostic apparatus, the probe frequency is set to $7.5 \sim 10 \mathrm{MHz}$. Patient supine, with a soft pillow in the patient's neck, to help patients head back Yang, the probe placed in the thyroid cartilage position, glide from top to bottom. Measurement of the distance from top to bottom, on the left and right thyroidal leaf length of the longitudinal section of scanning, scanning at the same time need to thyroid gland occupancy of the shape, size, edge and other parts were observed. Observe ultrasonography, the above parts of the sound halo, internal echo, or whether there is echo and calcification of the posterior wall. On the other hand, the neck appears enlarged lymph nodes were observed. When diagnosing a patient's condition, the doctor should encourage the patient to do shallow breathing as much as possible and prohibit the swallowing motion to ensure the clarity of the ultrasound image. After color ultrasound diagnosis, the patient receives a clinicopathological diagnosis. 
The diagnostic criteria of ultrasonography include benign and malignant lesions. The specific conditions of benign lesion are as follows: the halo around the nodules of the patient is relatively complete and there are multiple nodules by ultrasonography; the multiple nodules are presently in a regular state There is a clear edge of multiple nodules, internal echo more uniform, but obvious calcification, the internal blood flow is more abundant, showing a type I distribution. Specific conditions of malignant lesions: ultrasound findings showed solitary nodules, showing fine sand-like calcification, check the lesion internal echo, showing a heterogeneous trait, the patient's neck will be accompanied by lymph node metastasis.

According to color Doppler ultrasound tips, the blood flow was evaluated. The blood flow is divided into four levels, respectively, 0, I, II, III grade. Grade 0 blood flow status is mainly no indication of nodules within the presence of blood flow signals; I level prompted the presence of a small amount of blood flow signals, nodules within the presence of $<2$ blood flow signals; II level prompted the existence of the amount of blood flow signals, Nodules there are 2 to 4 points of blood flow signals, and prompt the existence of clear vessel wall; grade III prompted the presence of rich blood flow, nodular punctate blood flow $>4$ or prompted two clear wall vessels . Ultrasound imaging examination of benign and malignant lesions, the basis for the diagnosis of mass lesions based on the relevant research to develop the standard bricks, if the nodule score $\leq 3$ points is set to benign,> 4 points for the malignant lesions.

\section{Discussions}

Thyroid space-occupying lesions, also known as thyroid nodules, are clinically common endocrine system diseases. Thyroid is located below the thyroid cartilage, trachea on both sides of the central isthmus and the left and right sides of the two leaves, and sometimes up at the isthmus a cone-shaped leaves, through the fibrous tissue and thyroid levator muscle connected to the hyoid bone [2]. Due to its special anatomical location, in the past clinical work, its diagnosis and differential diagnosis have some difficulties, so it is easy to delay the treatment [3]. Thyroid lesions occupy the majority of benign lesions, but there may still be some malignant lesions, therefore, early diagnosis is crucial for the treatment of late. With the development of imaging, ultrasound technology has significantly improved. Color Doppler ultrasound can show the blood flow within the clumps, blood flow velocity, which is conducive to the identification of vascular lesions and non-vascular lesions. In addition, ultrasound examination of thyroid lesions can also understand the nature of the lesions at the blood vessels, which found shunt phenomenon and reflux. Compared with other examination methods, ultrasound has one of the biggest advantages of repeated examination, non-invasive, does not affect the patient's body, high safety, so as to better diagnose the patient. The study shows that the detection rate of malignant tumors ultrasound was $17.6 \%$, the detection rate of malignant tumors was $12.9 \%$, ultrasound has a certain accuracy. In summary, ultrasonic diagnosis has the advantages of noninvasive, reproducible, high recognition rate, thyroid lesions can be found early, and the accuracy rate can be used as preoperative imaging method of choice.

\section{Experimental Results}

Totally 77 patients with thyroid gland lesions were diagnosed by ultrasonography in 80 patients with confirmed thyroid gland lesions. Among them, 74 were benign lesions, 3 were malignant lesions, and the detection rate of thyroid-space lesions was $96.3 \%$. See Table 1. Postoperative pathological diagnosis found that 75 cases of benign lesions, 5 cases of malignant lesions. Ultrasound diagnosis and pathological diagnosis results compared to 1 case of benign lesions misdiagnosis, 2 cases of benign lesions missed diagnosis, 1 case of malignant lesions missed diagnosis. Ultrasound imaging features of benign lesions are mainly equal or mixed echo echo, nodular boundaries clear, regular, but poor blood supply, aspect ratio $<1$. Ultrasonic features of malignant nodules, hypoechoic state, nodular boundaries blurred, the shape showed irregular state, 
micro-calcification, rich in blood supply, aspect ratio $>1$. Pathological diagnosis of malignant lesions in 5 patients with papillary carcinoma (6.3\%), benign lesions in 6 cases of proliferative nodules $(7.5 \%)$, follicular adenoma in 7 patients (8.7\%), inflammatory nodules in 6 patients ( $7.5 \%)$, nodular goiter in 16 patients (20.0\%). Ultrasound diagnosis of malignant lesions in 3 patients with papillary carcinoma (3.8\%), benign lesions in nodular hyperplasia in 5 patients (6.3\%), follicular adenoma in 7 patients (8.7\%), inflammatory nodules. There were 6 patients (7.5\%) and 16 (20.0\%) patients with nodular goiter. There was no significant difference between the two diagnostic methods $(\mathrm{P}>0.05)[4]$.

Research reports show that ultrasound technology is constantly in the development process, the diagnosis of high accuracy, and color Doppler ultrasound diagnosis of high coincidence rate, the inspection time is shorter, less damage to the patient's body, the sensitivity of the test Degree and specificity higher. Previous grayscale ultrasound can only show the diameter of thyroid nodules, shape and echo. The use of color Doppler ultrasound can intuitively show the thyroid nodules and peripheral hemodynamic level, the judgment of benign and malignant tumors can provide an important reference. The clinical results show that the imaging features of the thyroid gland lesions diagnosed by two-dimensional and color Doppler ultrasound are mainly intra-nodular hypoechoic or non-uniform echogenicity, and show dot-like microcalcifications, nodule boundaries Showing fuzzy, nodules showed irregular arrangement of the shape, no sound halo, rich in blood supply, and the emergence of cervical nodular metastasis [5]. Combined with the clinical manifestations of this patient and the results of pathological examination, we found that color Doppler ultrasound detection of thyroid gland occupying lesions of clinical diagnosis and treatment of great significance. The relevant results show that the characteristics of benign lesions of the ultrasound images are mainly equal or mixed echo echo nodules clear boundaries, the rules of morphology, but poor blood supply, aspect ratio $<1$. Ultrasonic features of malignant nodules, hypoechoic state, nodular boundaries blurred, the shape showed irregular state, micro-calcification, rich in blood supply, aspect ratio $>1$. Color Doppler ultrasound examination of thyroid-space occupying lesions detection rate was $96.3 \%$, a higher detection rate. It can be seen from these points, color ultrasound diagnosis of thyroid lesions mainly observed nodules edge, nodular echo classification, calcification status of nodules, aspect ratio, nodule morphology and blood flow dynamics And other indicators, especially for hemodynamic indicators observed, you can find the body's blood flow direction, you can distinguish between arteries and veins. Correct response to the relevant properties of diseased vessels can be reflected on the reflux and shunt. These index data can provide important diagnostic evidence for clinical diagnosis. And this data show that the results of ultrasound diagnosis of nodular type and pathological diagnosis were compared, the difference was not statistically significant $(\mathrm{P}>0.05)$. This shows that the results of the two diagnostic methods are similar, and the diagnostic results of ultrasound are more accurate.

\section{Conclusions}

In short, preoperative thyroid gland lesions using color Doppler ultrasound diagnosis is conducive to the smooth operation and improves the clinical surgical treatment. Color Doppler ultrasound diagnosis is simple, timely, less damage, high diagnostic accuracy of ultrasound, which can provide important reference data for clinical diagnosis, clinical diagnosis of thyroid lesions have important clinical significance.

\section{References}

[1] Zhang Yao, Zhang Ying. Ultrasound elastography in the diagnosis of thyroid nodules disease value [J]. Clinical Medicine Engineering. 2016 (09). 62-67.

[2] Jiang Tao, Xu Lihua, Huang Bin. Ultrasound in the diagnosis of thyroid space-occupying lesions in the value of [J]. Modern Chinese Medicine. 2016 (10).55-56.

[3] Feng Smei, Liu Jiping. II diabetes mellitus combined with thyroid disease [J]. The world's latest 
medical information abstracts. 2016 (27).120-124.

[4] Li Kang, Yu Jianchun, Kang Weiming. Iodine intake and thyroid disease [J]. Chinese Journal of Endemiology. 2016 (03).99-100.

[5] Peng Liqin. Ultrasound diagnosis of thyroid space-occupying lesions of clinical value [J]. Contemporary Medicine. 2016 (08).67-69.

[6] Peng Li. Ultrasound diagnosis of thyroid disease in elderly population value and etiology [J]. Chinese community physicians. 2016 (01).55-56. 